\title{
Vasopressin, tissue-specific defects and the Brattleboro rat
}

from T.I. Bonner and M.J. Brownstein

Without the Brattleboro rat, we would be short of a good animal model in which to study the importance of the mammalian antidiuretic hormone, vasopressin. Brattleboro rats inherit diabetes insipidus as a recessive trait; homozygous animals excrete large volumes of dilute urine and suffer from extreme thirst, and they lack the normal content of vasopressin in their posterior pituitary. Had the genetic defect of the Brattleboro rat turned out to be a simple deletion of the vasopressin gene, matters would be simple but uninteresting. Several recent papers reveal a much more intriguing situation.

Vasopressin is synthesized in the cell bodies of neurones of the hypothalamic region of the brain in the form of a precursor which also contains two other peptides, neurophysin and a nameless glycopeptide. The precursor is packaged into vesicles and is enzymatically processed into its constituent peptides during the passage of the vesicles, within the axons of the neurones, to the posterior pituitary. The vesicular contents are released into blood vessels in the pituitary to act on peripheral target tissues. If the posterior pituitaries of normal animals are removed, they develop diabetes insipidus like the homozygous Brattleboro rat.

In attempting to define the genetic defect of the Brattleboro rat, Schmale and Richter have recently cloned a vasopressin gene from one such animal and have shown that it has a single-nucleotide deletion within the second of three exons ${ }^{1}$. We should note at the outset that it is a formal possibility that this deletion is not the controlling defect since it has neither been shown that the rat is homozygous for the deletion nor that it is present in more than one Brattleboro rat. Taken at face value, however, the deletion results in a frameshift which alters the amino acid sequence of one-third of the neurophysin, eliminates the arginine by which the glycopeptide is cleaved from it, completely alters the sequence of what would have been the normal glycopeptide and removes its glycosylation site, the normal termination codon. By removing 5 of the 13 cysteine residues normally present in the neurophysin, the frameshift results in a precursor that may be so drastically altered that it is no longer properly processed or transported. Schmale and Richter also speculate that the absence of a termination codon could impede translation of the mRNA by preventing ribosomes from disengaging from mRNA, a proposal which should be tested by deleting the termination codon of the normal gene and assaying its translation efficiency. The gene defect of the Brattleboro rat could account for the presence of small amounts of 'peptide $\mathrm{X}^{\prime}$ in Brattleboro hypothalami ${ }^{2}$. This peptide is similar in size to the normal vasopressin precursor and has vasopressinand neurophysin-like immunoreactivity, but is not glycosylated.

Although Schmale and Richter find equivalent amounts of vasopressin mRNA in Brattleboro and normal rats, suggesting that there is no defect in transcription, this result is disputed by Majzoub et al. who find that dehydrated Brattleboro rats have only 5 per cent of the hypothalamic vasopressin mRNA of similarly dehydrated Long Evans rats ${ }^{3}$. Since dehydration of the Long Evans rat results in a 20-fold increase in hypothalamic vasopressin mRNA, their conclusion is that the Brattleboro defect is transcriptional rather than translational.

Recently vasopressin has been detected in ovaries, adrenals (see references cited in refs 4 and 5) and sympathetic ganglia ${ }^{6}$ of normal rats. One might have predicted that the Brattleboro rat would have no vasopressin in its adrenal or ovary, just as it has none in its pituitary, but this seems not to be the case. On page 64 of this issue of Nature, Nussey and co-workers report that the adrenals of homozygous Brattleboro rats have a normal content of vasopressinlike material as measured by radioimmunoassay, and that the material has the same chromatographic properties as authentic vasopressin, though one might quibble over the chromatographic resolution ${ }^{4}$. Moreover, it is biologically active and its antidiuretic action is blocked by a specific vasopressin antagonist. Similarly, on page 61, Lim et al. show that the ovaries of Brattleboro rats contain as much vasopressin-like peptide as ovaries from Long Evans rats ${ }^{5}$. In the ovary, the peptide is confined to luteal cells; in the adrenal, it is present in chromaffin cells. Despite the fact that neither the adrenal nor the ovary vasopressin-like peptide has been purified and chemically characterized, it seems likely that they are both the same nonapeptide as is present in the posterior pituitary of normal animals. Thus, the defect in vasopressin production in the Brattleboro pituitary seems to be tissue specific; how the tissue-specificity can be reconciled with the gene defect is unclear.

One possible explanation is that there is a second gene for vasopressin or a closely related peptide and it is this gene that is expressed in the adrenal and ovary. There is a precedent for this suggestion in marsupials, which produce two vasopressin-like peptides ${ }^{7}$. The only datum relevant to the rat is a Southern blot of rat DNA using the whole rat vasopressin gene as probe ${ }^{1}$ but at such high stringency that even the closely related oxytocin gene was not detected. Thus, this experiment only excludes the presence of a second gene if it is nearly identical to the first.

A second possibility is that there is tissuespecific splicing of the vasopressin RNA which results in the substitution of an alternate exon for the defective second exon in the adrenal and the ovary. Both hypotheses predict that the size of the vasopressin mRNA in the adrenal and ovary would be different from that in the hypothalamus; characterization of these mRNAs will be critical.

It would be helpful to know whether immunoreactive neurophysin and glycoprotein are present in Brattleboro adrenals and ovaries. The observation of an immunoreactive glycopeptide in Brattleboro adrenals but not pituitary (E. Mezey, personal communication) is consistent both with the existence of a second gene that encodes a similar glycopeptide (the oxytocin gene does not) and with alternate splicing which retains the third exon encoding the glycopeptide. Examination of the rat and bovine gene sequences 1.8 indicates that an alternate second exon may be present in the large intron between the first and second exons (nucleotides 892-952 of the bovine sequence).

Despite preliminary evidence (the presence of the glycopeptide in Brattleboro adrenal) to the contrary, the vasopressin message in the adrenal and ovary of the Brattleboro rat may prove to be the same abnormal message that is found in the hypothalamus. If so, it would imply that the abnormal precursor gives rise to normal levels of vasopressin in the adrenal and ovary but not in the pituitary because of a tissue-specific post-translational defect. The most trivial explanation would be that Brattleboro hypothalamic neurones make vasopressin but it is released as rapidly as it is formed. Alternatively, cells in the adrenal and ovary may handle the abnormal precursor differently than neurones do. It may be possible to explore these possibilities with careful pulselabelling studies, but it seems that until we understand the roles of the various components of the vasopressin precursor, we shall not understand the Brattleboro rat's deficit.

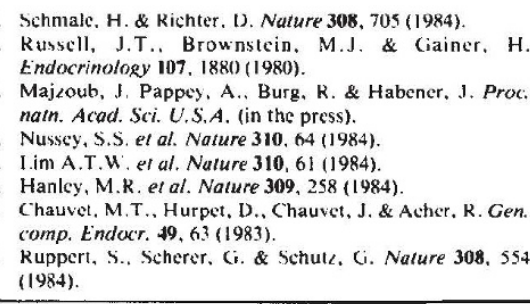
T.I. Bonner and M.J. Brownstein are in the Laboratory of Cell Biology, National Institute of Mental Health, Bethesda, Maryland 20205. 